\title{
Simulation of a carbon nanotube-based compliant parallel-guiding mechanism: A nanomechanical building block
}

\author{
Martin L. Culpepper \\ Christopher M. DiBiasio \\ Robert M. Panas \\ Spencer P. Magleby \\ magleby@byu.edu \\ Larry L. Howell \\ Ihowell@byu.edu
}

Follow this and additional works at: https://scholarsarchive.byu.edu/facpub

Part of the Mechanical Engineering Commons

\section{Original Publication Citation}

Culpepper, Martin L., Christopher M. Dibiasio, Robert M. Panas, Spencer Magleby, and Larry L. Howell. "Simulation of a carbon nanotube-based compliant parallel-guiding mechanism: A nanomechanical building block." Applied Physics Letters 89 (26).

\section{BYU ScholarsArchive Citation}

Culpepper, Martin L.; DiBiasio, Christopher M.; Panas, Robert M.; Magleby, Spencer P.; and Howell, Larry L., "Simulation of a carbon nanotube-based compliant parallel-guiding mechanism: A nanomechanical building block" (2006). Faculty Publications. 281.

https://scholarsarchive.byu.edu/facpub/281

This Peer-Reviewed Article is brought to you for free and open access by BYU ScholarsArchive. It has been accepted for inclusion in Faculty Publications by an authorized administrator of BYU ScholarsArchive. For more information, please contact ellen_amatangelo@byu.edu. 


\title{
Simulation of a carbon nanotube-based compliant parallel-guiding mechanism: A nanomechanical building block
}

\author{
Martin L. Culpepper, ${ }^{\text {a) }}$ Christopher M. DiBiasio, and Robert M. Panas \\ Department of Mechanical Engineering, Massachusetts Institute of Technology, Cambridge, \\ Massachusetts 02139 \\ Spencer Magleby and Larry L. Howell \\ Department of Mechanical Engineering, Brigham Young University, Provo, Utah 84602
}

(Received 4 August 2006; accepted 2 October 2006; published online 15 November 2006)

\begin{abstract}
The authors report the behavior of a nanoscale parallel-guiding mechanism wherein the compliant components are single-walled carbon nanotubes. Parallel-guiding mechanisms are often the building blocks of macro- and microscale mechanical systems. The authors present results that provide insight into the performance of a parallel-guiding mechanism for nanoscale devices. The device exhibits a range over $75 \%$ of the device size, i.e., $5.5 \mathrm{~nm}$, when actuated with $6.4 \mathrm{nN}$. Below $3.6 \mathrm{nN}$, displacements are due to bulk elastic bending of the nanotubes. Above $5.2 \mathrm{nN}$, displacements are governed by the hingelike bending of kinks in the nanotubes. van der Waals forces are shown to cause direction-dependent behavior. (c) 2006 American Institute of Physics.
\end{abstract}

[DOI: $10.1063 / 1.2388143]$

Parallel-guiding mechanisms ${ }^{1}$ (PGMs) are ubiquitous building blocks of micro- and macroscale mechanical systems. An illustration of a compliant PGM is shown in Fig. 1(a). The development of a nanoscale version of a compliant PGM would greatly expand the type and variety of nanomechanical devices that may be realized. The first step in this development is to examine possible embodiments and their characteristics. Toward this end, we present the simulated performance of a nanoscale PGM (nPGM) wherein two single-walled carbon nanotubes (SWCNTs) act as the motion guiding components.

As shown in Fig. 1(a), PGMs are one degree-of-freedom devices that consist of four parts. Part 1 is grounded and parts 2 and 4 deform elastically so as to guide the rigid coupler, part 3, through an arcuate path. This motion may be repeated through many cycles if the stresses within the compliant components do not exceed a critical failure stress. The use of compliant PGMs in precision and high-cycle mechanical systems predates $1989^{2}$ for microscale devices and $1937^{3}$ for macroscale devices.

PGMs may be used to create mechanical devices with arcuate or linear motion capabilities. For example, consider the microscale device in Fig. 1(b) that uses series combinations of "a" and "b" compliant PGMs to create sets 1 and 2 that are combined in parallel to guide the central component of the device along a linear path.

Carbon nanotubes (CNTs) are attractive for use in nPGMs due to their ability to deform elastically in excess of $40 \%$ strain. ${ }^{4}$ This would enable a CNT-based nPGM to operate over a range that could not be obtained with conventional materials. This property and other unique mechanical properties of CNTs have spurred interest in a variety of CNTbased nanomechanical machine components such as rotary bearings ${ }^{5-9}$ telescopic sliding bearings, ${ }^{10,11}$ and helical boltnut-type mechanical components. ${ }^{12-14}$ The nPGM is distinguished from those CNT-based components in its ability to

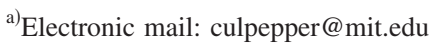

guide motion along a well-defined arc (for one PGM) or a straight line (for a series pair of PGMs).

We obtain insight on a specific nPGM via molecular simulation using the model shown in Fig. 1(a). The model consists of two parallel SWCNTs, parts 2 and 4, that are connected in parallel via part 1 (a structural ground) and part 3 (coupler). The ground and coupler are assumed to possess a high stiffness that warrants modeling them as rigid relative to the SWCNTs. The bottoms of the tubes are grounded and constrained at their tops by a rigid connection to the coupler by way of distance constraints. A force, $F$, is applied to the coupler in the $x$ direction. The tubes used in the simulations were defect-free. The idealized nature of this model is intentional as this provides a benchmark for future studies on the effect of defects, coupler compliance, etc. The characteristics of the CNTs are given in Table I.

In the molecular mechanics simulations, a Polak-Ribiere conjugate gradient algorithm was used to minimize the energy of the system until the RMS gradient was less than $1 \mathrm{cal} / \AA$ mol. Molecular dynamics simulations were carried out over $240 \mathrm{ps}$ using step increments of $1 \mathrm{fs}$. The simulations used an exp- 6 form of the van der Waals potential function with a well depth of $44 \mathrm{cal} / \mathrm{mol}$ and an equilibrium separation distance of $3.88 \AA$. The molecular dynamic simulations were set to run at a constant temperature that is infinitesimally larger than $0 \mathrm{~K}$ with a bath relaxation constant

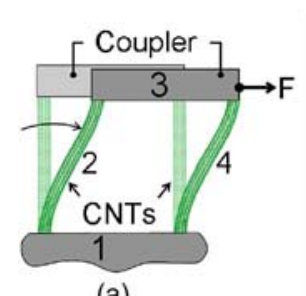

(a)

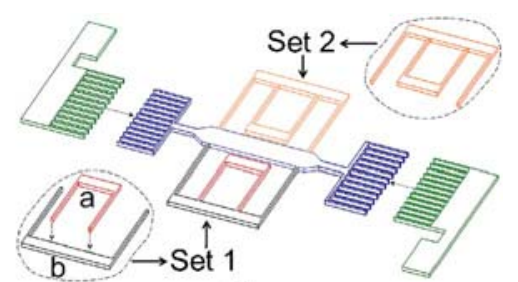

(b)
FIG. 1. (Color online) SWCNT-based parallel-guiding mechanism displaced from its equilibrium position (a) and linear bearing that uses series ( $a$ and $b$ ) and parallel (sets 1 and 2) arrangements of compliant PGMs (b). 
TABLE I. Parameters that describe the CNTs in the nPGM model.

\begin{tabular}{ll}
\hline \hline Tube length & $76.4 \AA$ \\
Tube-tube spacing & $76.4 \AA$ \\
Nominal diameter & $6.7 \AA$ \\
Chirality & $(5,5)$ \\
\hline
\end{tabular}

of $0.1 \mathrm{ps}$. These conditions are not meant to simulate practical function, rather they enable us to minimize the influence of thermal vibrations. These results provide an important base line for future studies on thermal vibration effects. The simulations revealed the two regions of operation shown in Figs. 2(a) and 2(b).

In the first region, the displacement of the device is governed by the bulk elastic deformation of the SWCNTs. This type of behavior is also observed in large-scale compliant PGMs. When the force on a nPGM surpasses a critical load, kinks form at the CNTs' ends in the order shown in Fig. 2(b). Region 2 occurs when each tube end has kinked, i.e., when there are four kinks. When the number of kinks is not zero (region 1) or not four (region 2), the device is in what we call a transition region. In region 2, the device's displacements are governed by the compliant, hingelike bending of the four kinks.

Figure 3 shows the results in graphical form. In Fig. 3, "M" denotes results from molecular mechanics simulations, "D" denotes results from molecular dynamics data, and " $L$ " and " $U$ " indicate if data were obtained during loading or unloading of the nPGM. During loading, the coupler travels via points $A-B-C-D-E$ and then passes through points $E-D-F-G-A$ on unloading. Figure 3(a) shows the trajectory of the coupler centroid, $x_{c}$ [the coupler centroid is located at the geometric center of part 3 in Fig. 1(a)]. During region 1 behavior, we observe coupler rotations that are less than $4^{\circ}$, matching with Jones' observations of coupler rotation in macroscale PGMs. ${ }^{15}$ During the transition from region 1 to region 2 behavior, we observe that the kinking induced motions in $x_{c}$ and $y_{c}$ are less than $20 \mathrm{pm}$. As such, the centroid appears to follow a smooth trajectory in Fig. 3(a).

The difference between the loading and unloading curves shown in Figs. 3(b)-3(d) is attributed to two phenomena: (1) van der Waals interactions and (2) the geometric nonlinearity associated with the buckled tube geometry. We will first discuss what determines the shape of the loading curve. The equilibrium distance for non-bonded carbon atoms is $3.8 \AA$, or roughly half of the $(5,5)$ SWCNT's diameter. This is important as it means that some nonbonded atoms within any given cross section of the tube are separated by less than the equilibrium spacing of graphite, $\sim 3.8 \AA$, and therefore these atoms will repulse each other. Figures 4(a) and 4(b) show the cross section of our $(5,5)$ tube at a load corresponding to point $B$ in Fig. 3. Any nonbonded atom within the circle shown in Fig. 4(a) will experience a

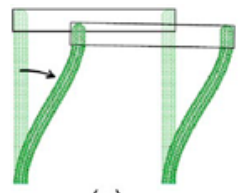

(a)

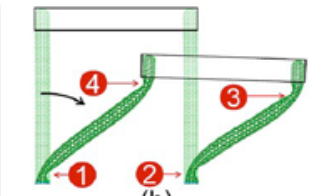

(b)
FIG. 2. (Color online) Region 1: non-buckled region of operation (a); region

2: buckled region of operation (b).
Downloaded 13 Feb 2009 to 128.187 .0 .164 . Redistribution subject to AIP license or copyright; see http://apl.aip.org/apl/copyright.
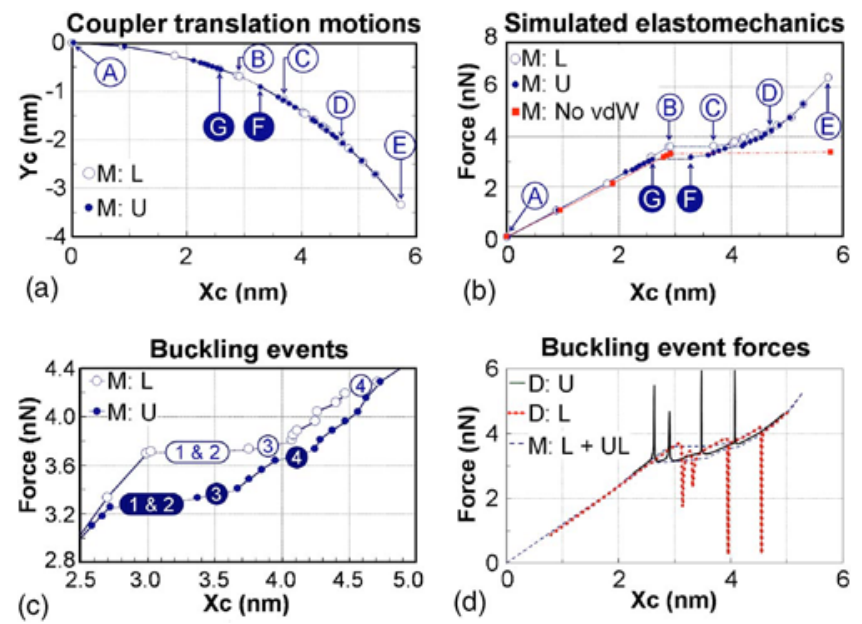

FIG. 3. (Color online) Kinematics (a), elastomechanic response (b), close-up of the transition between regions 1 and 2 (c), and dynamic simulation results superimposed on molecular mechanics results (d).

repulsive force with respect to the atom at the center of this zone. The atom clearly repulses two other adjacent but nonbonded atoms in the cross section. The lines in Fig. 4(b) represent all repulsive forces between nonbonded atoms in this cross section. Figure 4(c) shows the difference between the buckled and nonbuckled cross sections at various states of loading.

These inter-cross-section repulsive forces are resisted by the $\mathrm{C}-\mathrm{C}$ bond forces within the cross section. The repulsive forces grow stronger as the cross section ovalizes with increased load as shown in Fig. 4(c). Our simulations and the vector sum of calculated forces between all atoms in the cross section show that repulsive forces dominate the attractive forces during region 1 operation. Table II shows the calculated repulsive force between the upper and lower halves of the cross sections in Fig. 4(c).

The "truss-like" arrangement of forces in Fig. 4(b) acts to resist continued ovalization of the tube. This resistance leads to a prebuckled stiffness that is higher than one would find in the absence of van der Waals forces. This van der Waals interaction effect would be more pronounced for smaller radius tubes as adjacent nonbonded atoms would be closer. As such, the elastomechanic response of a CNT-based nPGM will be diameter specific. The effect on our nPGM may be observed in Fig. 3(b) by comparing a loading curve (M: L) that captures van der Waals interactions with the loading curve (M: no vdW) that does not include these effects. The van der Waals interactions lead to higher device stiffness (slope) in region 1 and a higher critical buckling load at which the tubes lose bending stiffness. ${ }^{16}$

We now describe the reason for the shape of the unloading curve. At and beyond point $D$, all of the tube ends have
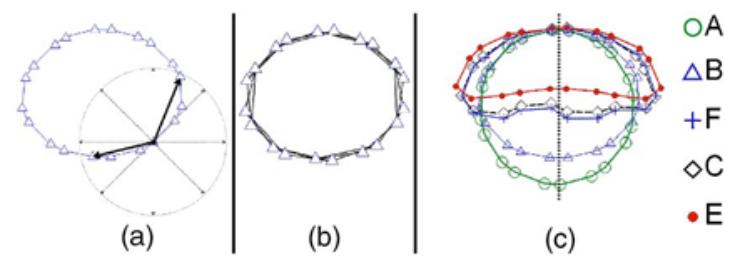

(c)

FIG. 4. (Color online) Zone surrounding an atom where van der Waals forces are repulsive (a), repulsive interactions between nonbonded atoms in cross-section $B(\mathrm{~b})$, and cross sections of kink 2 at different loads (c). 
TABLE II. Magnitude of repulsive force in a $(5,5)$ nPGM cross section.

\begin{tabular}{llllll}
\hline \hline & $A$ & $B$ & $C$ & $E$ & $F$ \\
\hline Force $(\mathrm{nN})$ & 3.8 & 4.2 & 7.1 & 15.3 & 6.0 \\
\hline \hline
\end{tabular}

buckled and the loading and unloading curves coincide. When the load is reduced below that of point $D$, it is not energetically preferential for the kinks to immediately revert back to their prebuckled circular cross sections. The combination of the tube end geometries and the lower bending stiffness of the buckled cross sections causes the unloading curve to follow a path that differs from the loading curve. There is precedence for this in that Chang et al. ${ }^{17}$ have shown that van der Waals interactions cause directiondependent elastomechanic behavior in axially buckled CNTs. As unloading continues, it eventually becomes energetically preferential for the kinks to revert back to a prebuckled cross section.

Given the different shapes of the load-unload curves, it is important to understand how the area beneath them can be equivalent. Figure 3(d) shows the results of molecular mechanics simulations $(\mathrm{M}: \mathrm{L}+\mathrm{UL})$ superimposed on molecular dynamics simulations during loading (D: L) and unloading (D: U). The molecular dynamics simulations enable us to capture the behavior of the mechanism during buckling. On loading, the force required to maintain position $x_{c}$ decreases sharply when a kink is formed. On unloading, the force required to hold the position increases when a buckled cross section reverts to a prebuckled shape. During these events, the potential energy stored within the kinks' bonds and the energy stored via van der Waals interactions are released into the structure of the tube and a sharp increase in load is experienced. Figure 3(d) shows these as "dips" and "spikes" during the loading/unloading process.

The net result of the dips and spikes is an equivalent area beneath the loading and unloading curves. Therefore the work done on the mechanism during loading and the work done by the mechanism during unloading would be equal. Our simulations show that the areas differ by less than $1 \%$ which is less than the accuracy of the simulations. This is important as these molecular mechanics simulations are based on a model wherein energy is conserved. This assumption is appropriate for this benchmarking study given the small dissipative forces observed during the bending deformation of CNTs. ${ }^{7,18}$

With respect to structural failure, we note that nonreversible bond reconfiguration, i.e., plastic deformation, over this range of operation is not expected. The smallest radius of curvature in the kinks is never less than $0.76 \mathrm{~nm}$; thus, no irreversible $s p 2$ to $s p 3$ bond changes should occur. ${ }^{19,20}$ The maximum strained bond length in any simulation is $1.6 \AA$, which is less than the bond failure length, between 1.7 and $1.9 \AA$, that is commonly used. ${ }^{21-24}$

The results in this letter provide insight into the types of behavior (buckled versus nonbuckled), the reason for these behaviors, and the performance characteristics (e.g., force stroke) one could expect from nPGMs that use SWCNTs. The results indicate that these devices have force and displacement ranges that are within the reach of nanoscale actuators. Present efforts are focused on obtaining a better understanding of how the nPGM's behavior changes with diameter, chirality, defects, length to diameter ratio, and number of nested tubes when multiwalled tubes are used.

${ }^{1}$ J. M. Derderian, L. L. Howell, M. D. Murphy, S. M. Lyon, and S. D. Pack, Proceedings of the 1996 ASME Mechanisms Conference 96-DETC/ MECH 1208 (2006).

${ }^{2}$ W. C. Tang, T. C. H. Nguyen, and R. T. Howe, Sens. Actuators 20, 25 (1989).

${ }^{3}$ R. S. Clay, J. R. Microsc. Soc. 57, 1 (1937).

${ }^{4}$ J. Lu and L. Zhang, Comput. Mater. Sci. 35, 432 (2006).

${ }^{5}$ B. Bourlon, D. C. Flattli, C. Milo, L. Forro, and A. Bachtold, Nano Lett. 4, 709 (2004).

${ }^{6}$ S. Zhang, W. K. Liu, and R. Ruoff, Nano Lett. 4, 293 (2004).

${ }^{7}$ A. M. Fennimore, T. D. Yuzvinksly, W. Han, M. S. Fuhrer, J. Cumings, and A. Zettle, Nature (London) 424, 408 (2003).

${ }^{8}$ J. C. Meyer, M. Paillet, and S. Roth, Science 309, 1539 (2005).

${ }^{9}$ S. J. Papadakis, A. R. Hall, P. A. Williams, L. Vicci, M. R. Falvo, R. Superfine, and S. Washburn, Phys. Rev. Lett. 93, 146101 (2004).

${ }^{10}$ J. Cumings and A. Zettl, Science 289, 602 (2000).

${ }^{11}$ M. Yu, B. I. Yakobson, and R. S. Ruoff, J. Phys. Chem. B 104, 8764 (2000).

${ }^{12}$ R. Saito, R. Matsuo, T. Kimura, G. Dresselhaus, and M. S. Dresselhaus, Chem. Phys. Lett. 348, 187 (2001).

${ }^{13}$ Y. E. Lozovik, A. V. Monogin, and A. M. Popov, Phys. Lett. A 313, 112 (2003).

${ }^{14}$ A. V. Belikov, Y. E. Lozovika, A. G. Nikolaev, and A. M. Popov, Chem. Phys. Lett. 385, 72 (2004).

${ }^{15}$ R. V. Jones, Instruments and Experiences (Wiley, New York, 1988), p. 106.

${ }^{16}$ L. G. Brazier, Proc. R. Soc. London, Ser. A 116, 104 (1927).

${ }^{17}$ T. Chang, J. Hou, and X. Guo, Appl. Phys. Lett. 88, 211906 (2006).

${ }^{18}$ A. Pantano, D. M. Parks, and M. C. Boyce, J. Mech. Phys. Solids 52, 789 (2004).

${ }^{19}$ A. Maiti, Chem. Phys. Lett. 331, 21 (2000).

${ }^{20}$ T. W. Ebbesen and T. Takada, Carbon 33, 973 (1995).

${ }^{21}$ M. Huhtala, A. V. Krasheninnikov, J. Aittoniemi, S. J. Stuart, K. Nordlund, and K. Kaski, Phys. Rev. B 70, 045404 (2004).

${ }^{22}$ Y. Xia, M. Zhaom, Y. Ma, M. Ting, X. Liu, P. Liu, and L. Mei, Phys. Rev. B 65, 155415 (2002).

${ }^{23}$ T. Dumitrica, T. Belytschko, and B. I. Yakobson, J. Chem. Phys. 118, 9485 (2003).

${ }^{24}$ Q. Lu and B. Bhattacharya, Eng. Fract. Mech. 72, 2037 (2005). 